\title{
A 0.1-23-GHz SiGe BiCMOS Analog Multiplier and Mixer Based on Attenuation-Compensation Technique
}

\author{
Ming-Da Tsai, Chin-Shen Lin, Chi-Hsueh Wang, Chun-Hsien Lien and Huei Wang \\ Dept. of Electrical Engineering and Graduate Institute of Communication Engineering, \\ National Taiwan University, Taipei, Taiwan
}

\begin{abstract}
A SiGe BiCMOS broadband mixer and analog multiplier using $L C$ ladder matching networks and attenuation-compensation method were first proposed. This broadband mixer achieves measured conversion gain of $19 \mathrm{~dB}$ with a 3-dB bandwidth from 0.1 to $23 \mathrm{GHz}$. The mixer was fabricated in a commercial $0.35-\mu \mathrm{m}$ SiGe BiCMOS technology and demonstrated the highest gain-bandwidth product of operation among previously reported MMIC mixers. As an analog multiplier, the measured sensitivity is better than $3000 \mathrm{~V} / \mathrm{W}$ from 0.1 to 25 GHz with $50-\Omega$ output impedance. The chip area is $1.5 \times$ $\mathrm{mm}^{2}$ including testing pad. The performance of this circuit represents state-of-the-art result of the MMIC broadband mixers using standard silicon-based technologies.

Index Terms - Monolithic microwave integrated circuits, heterojunction bipolar transistors, silicon, broadband communication, mixers.
\end{abstract}

\section{I . INTRODUCTION}

The Gilbert-cell multipliers and mixers have been demonstrated with various monolithic microwave integrated circuit (MMIC) technologies [1]-[16]. Record mixer RF bandwidth has been demonstrated using $0.1-\mu \mathrm{m}$ InP high electron-mobility transistor (HEMT) technology with low gain [2]. With high $f_{T}(70 \mathrm{GHz})$, the InP HBT-based analog mixer has demonstrated a record gain-bandwidth product (GBP) of $116.4 \mathrm{GHz}$ [19]. The highest operation frequency of reported broadband active mixer.was demonstrated using a $f_{\max }$ of $128 \mathrm{GHz}$ SiGe-HBT process, but it fall short of providing operation frequency below $12 \mathrm{GHz}$ [10]. With broadband input transmission-line matching networks, medium conversion gain of mixer from 1 to $17 \mathrm{GHz}$ in InGaP-GaAs HBT technology has been presented in [6].

In this study, we demonstrated a wide-bandwidth 0.1-23-GHz SiGe BiCMOS analog multiplier and mixer. This active mixer is based on the Gilbert transconductance multiplier cell. Although the $f_{\text {MAx }}$ of the device used in the design is about $57 \mathrm{GHz}$, significantly lower than that of the GaAs, SiGe, and InP HBT device, our LC ladder matching networks and attenuation-compensation method resulted in high gain and wide bandwidth for this chip. The MMIC chip without RF, LO, IF amplifiers also shows high frequency-conversion performance compared with those of GaAs, SiGe, and InP HBT analog multipliers and mixers. Fig. 1 summarizes published wide-band mixer integrated-circuit (IC) gain and bandwidth performance.
Our SiGe BiCMOS MMIC achieves a measured conversion gain of $19 \mathrm{~dB}$ and 3-dB bandwidth from 0.1 to $23 \mathrm{GHz}$ and, thus, even without a pre- or post-amplifier, gives the highest gain-bandwidth product of $204 \mathrm{GHz}$ among the reported SiGe, InP and GaAs-based HBT analog active mixer.

\begin{tabular}{|c|c|c|c|c|c|c|}
\hline Process & $\begin{array}{l}f, / / 2-1 \\
(\mathrm{GHz})\end{array}$ & Design Topology & $\begin{array}{l}\begin{array}{l}\text { Gain } \\
\text { (dB) }\end{array} \\
\end{array}$ & $\begin{array}{c}\text { Bandwidith } \\
\text { (GHz) }\end{array}$ & $\begin{array}{c}\mathrm{GBP} \\
(\mathrm{GH} 2)\end{array}$ & Ref. \\
\hline GaAs HBT & 90770 & Gilber Cell & 5 & $D C$ to 20 & 35.5 & III \\
\hline InP HEMT & $155 \%-$ & Distribuled mixer & .5 & DC to 38 & 21.4 & 121 \\
\hline InP HBT & $90 / 70$ & $\begin{array}{l}\text { Gilber cell with Lo drive and } \\
\text { IF buffer }\end{array}$ & 6 & $D C$ to 15 & 8 & [3] \\
\hline GaAs HBT & $40 / 40$ & $\begin{array}{l}\text { Gilber cell winh TL-matching } \\
\text { network }\end{array}$ & 9.3 & 1 to 17 & 45 & [6] \\
\hline GaAs HBT & - & Gillert dicromixer & 11 & $D C$ to 8 & 28.4 & [9] \\
\hline SiBT & $20 \%$ & Gilber Cell & is & $D C 108$ & 45 & [11] \\
\hline $\mathrm{SiBN}$ & $10 / 20$ & Gilber Coll & 16 & $D C 106$ & 37.8 & [13] \\
\hline SiGe HBT & 8097 & Gilben Cell & 5.9 & 21030 & $5 s .2$ & [14] \\
\hline $\mathrm{SiGe} \mathrm{HBT}$ & 80997 & Gilbert Cell & 10 & 1020 & 60 & [15] \\
\hline InP HBT & $70 / 160$ & 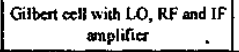 & is & DC to 20 & 116.4 & [16] \\
\hline $\begin{array}{c}\mathrm{SiGe} \\
\mathrm{BiCMOS}\end{array}$ & $80 / 57$ & $\begin{array}{l}\text { Gibert Cell with attenuation } \\
\text { compensation and LC ladder } \\
\text { matching network }\end{array}$ & 19 & 0.11023 & 204 & $\begin{array}{l}\text { This } \\
\text { Work }\end{array}$ \\
\hline
\end{tabular}

Fig. 1. Comparison of monolithic integrated mixers in various MMIC technologies

\section{MMIC PROCESS}

The broadband mixer was designed using TSMC commercial $0.35-\mu \mathrm{m}$ 3P3M SiGe BiCMOS technology, which provides three poly layers for the emitters and bases of the SiGe heter-junction bipolar transistor (HBT) and the gates of the complementary $\cdot \mathrm{MOS}$ (CMOS) transistors; three metal layers for inter-connection. The HBT manufactured in this technology offer cut-off frequency of $80 \mathrm{GHz}$, maximum oscillation frequency of $57 \mathrm{GHz}$. Unlike the high-resistivity substrate of SiGe HBT process, the SiGe BiCMOS process with lowresistivity substrate provides monolithic inductors with quality factor below 10. A MIM capacitor of $1 \mathrm{fF} / \mathrm{mm}^{2}$ has been developed using oxide inter-metal dielectric. Polysilicon resistors, with several $\Omega / \bullet$ and $k \Omega / \bullet$, are provided by choosing the individual dose of ion-implantation separately form the gate electron doping process. 


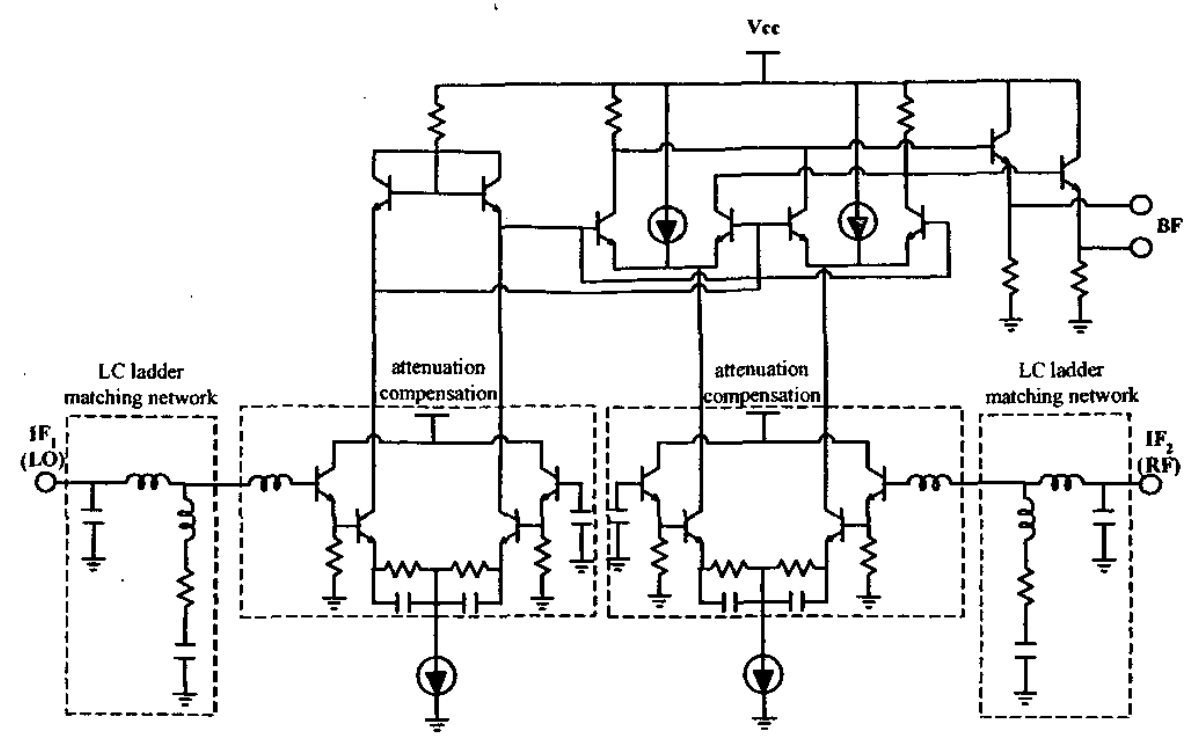

Fig. 2. Circuit schematic of the SiGe BiCMOS broadband mixer/multiplier

\section{CiRCuIt DESIGN}

The circuit schematic of this broadband mixer/multiplier is shown in Fig. 2. The circuit can be divided into the: 1) predistortion circuit; 2) dc-bias circuit; 3) output buffer; 4) Gilbert cell core; 5) input LC Iadder matching network; 6) attenuation-compensation circuit. The Gilbert cell configuration is selected for the double balanced 'implementation which offers high conversion gain and improved spur performance in a very compact size [1]. The RF signal enters a lower amplifier formed by the emitter coupled pair. The LO signal enters an upper cross coupled quad of devices through the predistortion circuit.

In order to improve the bandwidth, wideband impedance match is necessary for reducing signal reflection. Wideband impedance match can be achieved by equivalent $50-\Omega$ resistors shunted with the high input impedances of the input transistors in previous existing designs [1], but the capacitive-looking impedance of the emitter couple pairs (ECPs) at upper band edge will degrade the return loss [6]. Moreover, the shunt resistor deteriorates the conversion gain at high frequency. Broadband analog multiplier/mixer adopted transmission-line matching networks achieved better conversion gain and input return loss simultaneously than that in equivalent shunted $50-\Omega$ resistors [5].

However, the base parasitic resistance $\left(R_{\mathrm{B}}\right)$ of HBT transistors is generally larger than that of FETs depends on the transistor size [18]. The base resistance $\left(R_{\mathrm{B}}\right)$ increases the loss of the artificial transmission line formed by the LC ladder matching network. Moreover, the monolithic inductors in standard silicon substrate suffer more loss than that in GaAs or InP technology. These energy losses will degrade the overall performance of wideband mixer and multiplier, e.g. bandwidth and conversion gain, etc. In order to promote to high frequency performance in standard silicon technology, the attenuation-compensation method is adopted to overcome these obstacles.

The attenuation-compensation network consists of Darlington (CC-CE) configuration, and the LC ladder matching networks, as shown in Fig. 2, are adopted to implement the wideband impedance matching which is generally used in distributed circuits. The matching network is loaded by the input equivalent capacitance of attenuation-compensation network and is terminated in its characteristic impedance at the end with spiral inductors to perform an artificial transmission line. All the passive elements of capacitors and inductors are simulated by the full-wave simulation tool [17] to predict the high frequency effects. Fig. 3 shows the chip micrograph with a chip size of $1.5 \times 1 \mathrm{~mm}^{2}$.

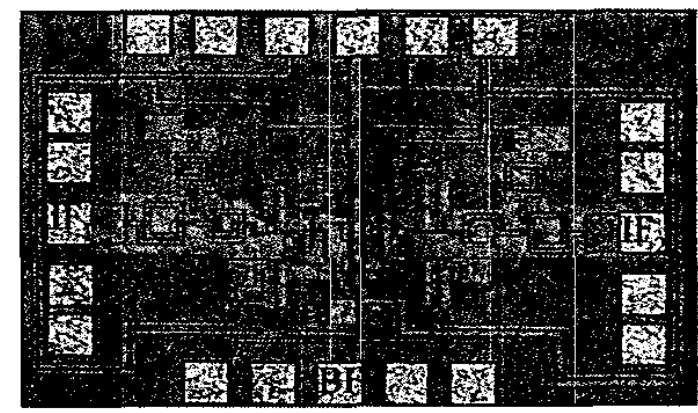

Fig. 3. Chip micrograph (size: $1.5 \mathrm{~mm} \times 1 \mathrm{~mm}$ ) 


\section{MEASUREMENT RESULTS}

The mixer characteristics of the chip were measured using on-wafer probing on $\mathrm{IF}_{1}$ as $\mathrm{LO}$ input port and $\mathrm{IF}_{2}$ as $\mathrm{RF}$ input ports with ground-signal-ground (GSG) probes. While measuring the multiplier characteristics, the input signals were fed from the IF pads and the output was extracted from the BF pad. This chip was measured using wire bonding of output pads as the output port (indicated as BF in Fig. 3). The output was then connected to a spectrum analyzer through dc-blocking capacitors. Fig. 4 shows the conversion gain as a function of LO power with RF frequency of $10 \mathrm{GHz}$ and IF frequency of $10 \mathrm{MHz}$. It shows that the conversion gain almost saturated approximately $0 \mathrm{dBm}$ of $L O$ power. The conversion gain versus the RF frequency is shown in Fig. 5 with both RF and LO ports swept in frequency up to $30 \mathrm{GHz}$, a fixed IF frequency of $10 \mathrm{MHz}$, and LO power of $-1 \mathrm{dBm}$. The conversion gain is approximately $19 \mathrm{~dB}$ with a 3-dB bandwidth of $23 \mathrm{GHz}$ from 0.1 to 23 GHz.

The multiplier characteristics were measured with the same setup as the mixer measurement. The output voltage versus the IF frequency is shown in Fig. 6, with two IF ports swept in frequency from 0.1 to $30 \mathrm{GHz}$, a fixed baseband frequency (BF) of $10 \mathrm{MHz}$, and $\mathrm{IF}$ power of $-20 \mathrm{dBm}$. The sensitivity, which is the ratio of $\mathrm{BF}$ output voltage to total IF input power, was better than $3000 \mathrm{~V} / \mathrm{W}$ with a bandwidth of $0.1-25 \mathrm{GHz}$. Total power consumption is $145 \mathrm{~mW}$.

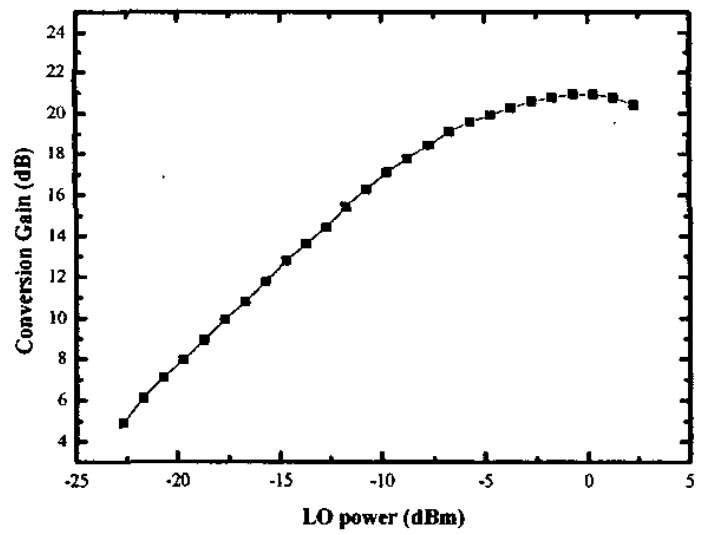

Fig. 4. The measured conversion gain versus $L O$ input power at $\mathrm{RF}$ frequency of $10 \mathrm{GHz}$

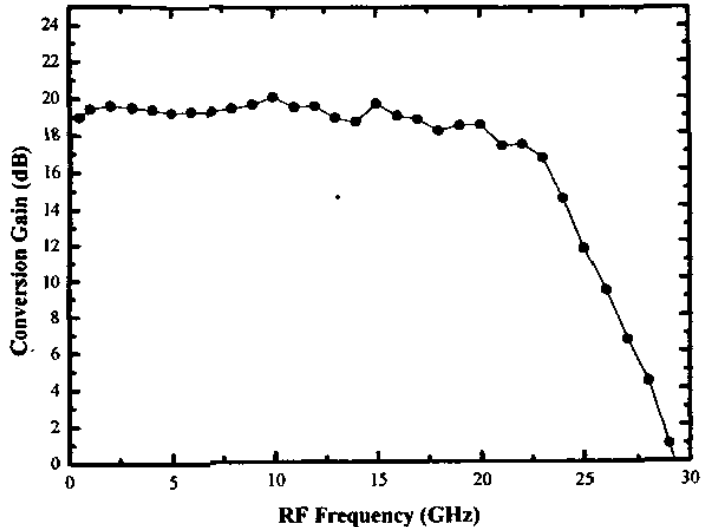

Fig. 5. Measured conversion gain versus RF frequency. The IF frequency $10 \mathrm{MHz}$, LO power is $0 \mathrm{dBm}$.

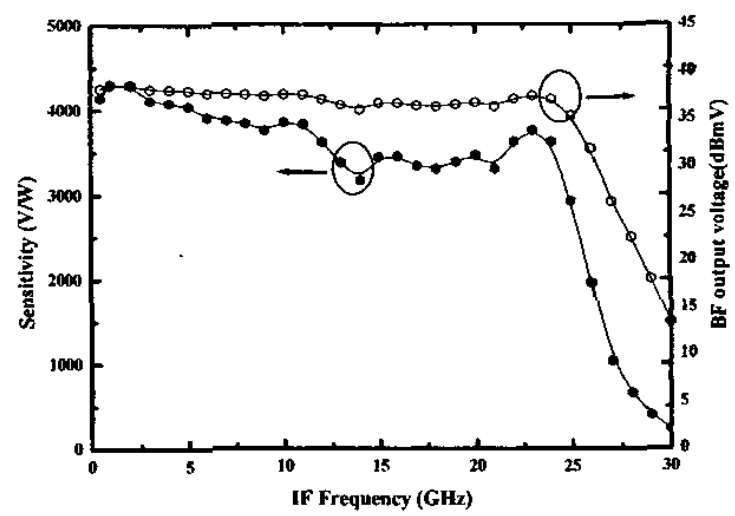

Fig. 6. Measured BF output voltage with IF power of $-20 \mathrm{dBm}$ and sensitivity from 0 to $30 \mathrm{GHz}$

\section{CONCLUSION}

An active broadband analog multiplier and mixer in commercial 0.35- $\mu \mathrm{m}$ SiGe BiCMOS technology has been presented. The mixer sets state-of-the-art for monolithic integrated mixers realized in standard silicon technologies. The LC ladder matching networks are used to achieve broadband impedance matching, and the attenuation-compensation method is also adopted to promote the conversion gain and bandwidth. A high conversion gain of $19 \mathrm{~dB}$ is achieved with a 3-dB bandwidth of 0.1-23 GHz. The highest gain-bandwidth product of $204 \mathrm{GHz}$ among the reported SiGe, InP and GaAs-based HBT analog active mixer is also achieved. Since the broadband mixer/multiplier was fabricated in standard silicon technology, it can be easily integrated with other $\mathrm{Si}$-based front-end circuits without requiring any post-processing steps. 


\section{ACKNOWLEDGEMENT}

This work is supported in part by National Science Council (NSC 92-2213-E-002-069 and NSC 92-2219-E-002-001) and Research Excellence Program funded by Department of Education of Republic of China (ME-89-E-FA06-2-4-6). The chip is fabricated by Taiwan Semiconductor Manufacturing Company (TSMC) through the Chip Implementation Center (CIC), Taiwan, ROC. The authors would like to thank Dr. Kun-You Lin of NTU and Dr. Chio-Te Li of ASIAA for the help of chip testing.

\section{REFERENCES}

[1] K. Osafune and Y. Yamauchi, "20-GHz 5-dB-gain analog multipliers with AIGaAs/GaAs HBTs," IEEE Trans. Microwave Theory Tech., vol. 42, pp. 518-520, Mar. 1994.

[2] Y. Imai, S. Kimura, Y. Umeda, and T. Enoki, "DC to 38-GHz distributed analog multiplier using InP HEMT's," in IEEE Microwave Guided Wave Lett., vol. 4, pp. 399-401, Dec. 1994.

[3] L. M. Burns, J. F. Jensen, W. E. Stanchina, R. A. Metzger and Y. K. Allen, "DC-to-Ku-band MMIC InP HBT double-balanced active mixer" in IEEE Int. Solid-State Circuits Conf. Dig., San Francisco, CA, 1991, pp. 124-125.

[4] J. Glenn, M. Case, D. Harame, B. Meyerson, and R. Poisson, "12-GHz Gilbert mixers using a manufacturable $\mathrm{Si} / \mathrm{Si}-\mathrm{Ge}$ epitaxial-base bipolar technology," in Proc. IEEE Bipolar/BiCMOS Circuits Technol. Meeting, Minneapolis, MN, 1998, pp. 186-189.

[5] H. Wang, "A $1-V$ multi-gigahertz RF mixer core in $0.5-\mu \mathrm{m}$ CMOS," in IEEE Int. Solid-State Cincuits Conf. Dig., San Francisco, CA, 1998, pp. 370-371.

[6] B. Tzeng, C. H. Lien, H. Wang Y. C. Wang, P. C. Chao, and C. H. Chen, "A 1-17-GHz InGaP.GaAs HBT MMIC analog multiplier and mixer with broad-band input-matching networks," IEEE Trans. Microwave Theory Tech., vol. 50, pp. 2564-2568, Nov. 2002

[7] I. Gresham and A. Jenkins, "a low-noise broadband $\mathrm{SiGe}$ mixer for 24-GHz ultra-wideband automotive applications," in IEEE RAWCON Proc., pp. 361-364, 2003

[8] A. Y. Umeda, C. T. Matsuno, A. K. Oki, G. S. Dow, K.W. Kobayashi, D. K. Umemoto, and M. E. Kim, "A monolithic GaAs HBT upconverter," in IEEE Microwaves and Millimeter-Wave Monolithic Circuits Symp. Dig., Dallas, TX, 1990, pp. 77-80.

[9] C. C. Meng S. S. Lu, M.H. Chiang and H. C. Chen, "DC to $8 \mathrm{GHz} \| \mathrm{dB}$ gain Gilbert micromixer using GaInP/GaAs HBT technology," Electronics Lett., vol. 39, pp. 637-638, April 2003.

[10] S. Hackl, J. Bock, M. Wurzer, and A. L. Scholtz, " $40 \mathrm{GHz}$ monolithic integrated mixer in SiGe bipolar technology," in IEEE MTT-S Int. Microwave Symp. Dig., Seattle, 2002, vol. 2, pp. 1241-1244.

[11] P. Weger, G. Schuites, L. Treitinger, E. Bertagnolli, and K. Ehinger, "Gilbert multiplier as an active mixer with conversion gain bandwidth of up to $17 \mathrm{GHz}, "$ Electronics Lett., vol. 27, no. 7, pp. 570-571, Mar. 1998.

[12] K. W. Kobayashi, L. T. Tran, S. Bui, A. K. Oki, D. C. Streit and $M$. Rosen, "InAlAs/InGaAs HBT X-band double-balanced upconverter," IEEE J. Solid-State Circuits, vol. 29, pp. 1238-1243, Oct. 1994.

[13] J. Wholey, I. Kipnis, and C. Snapp, "Silicon bipolar double balanced active mixer MMIC's for RF and microwave applications up to $6 \mathrm{GHz}$," in IEEE Microwaves and
Millimeter-Wave Monolithic Circuits Symp. Dig., Long Beach, CA, 1989, pp. 133-137.

[14] M. Wurzer, T. F. Meister, S. Hackl, H. Knapp, L. Treitinger, " $30 \mathrm{GHz}$ active mixer in Si/SiGe bipolar technology," in IEEE APMC Proc., pp.780-782, Dec. 2000

[15] S. Hackl, M. Wurzer, J. Bock, T. F. Meister, H. Knapp, K. Aufinger, L. Treitinger, A. L. Scholtz, "Low-noise, low-power monolithically integrated active $20 \mathrm{GHz}$ mixer in SiGe technology," Electmnics Lett., vol. 37, № 1, pp.36-37, Jan. 2001

[16] K. W. Kobayashi, R. M. Desrosiers, A. K. Oki, and D.C. Streit, "A DC-20-GHz InP HBT balanced analog multiplier for high-data-rate direct-digital modulation and fiber-optic receiver application," IEEE Trans. Microwave Theory Tech. vol, 48, pp. 194-202, Feb. 2000.

[17] Sonnet User's Manual, Sonnet Software Inc., Liverpool, NY, 1998.

[18] K. W. Kobayashi, R. Esfandiari and A. K. Oki, "A novel HBT distributed amplifier design topology based on attenuation compensation techniques," IEEE Trans. Microwave Theory Tech., vol. 42, pp. 2583-2589, Dec. 1994. 